\title{
Hybrid metal-dielectric, slow wave structure with magnetic coupling and compensation
}

\author{
A. V. Smirnov, ${ }^{1, a}$ and E. Savin ${ }^{1,2, b}$ \\ ${ }^{1}$ RadiaBeam Systems LLC, 1713 Stewart St., Santa Monica, CA 90404, US \\ ${ }^{2}$ National Research Nuclear University “MEPhI”, Moscow, 115409, Russian Federation
}

A number of electron beam vacuum devices such as small radiofrequency (RF) linear accelerators (linacs) and microwave traveling wave tubes (TWTs) utilize slow wave structures which are usually rather complicated in production and may require multi-step brazing and time consuming tuning. Fabrication of these devices becomes challenging at centimeter wavelengths, at large number of cells, and when a series or mass production of such structures is required. A hybrid, metaldielectric, periodic structure for low gradient, low beam current applications is introduced here as a modification of Andreev's disk-and-washer (DaW) structure. Compensated type of coupling between even and odd TE01 modes in the novel structure results in negative group velocity with absolute values as high as $0.1 \mathrm{c}-0.2 \mathrm{c}$ demonstrated in simulations. Sensitivity to material imperfections and electrodynamic parameters of the disk-and-ring $(\mathrm{DaR})$ structure are considered numerically using a single cell model.

${ }^{a}$ Corresponding author: asmirnov@ radiabeam.com

b On leave from NRNU "MEPhI” for RadiaBeam Systems, LLC. 


\section{Introduction}

Industrial linac systems are employed in a wide variety of applications, from radiography to sterilization. In general, such a conventional system consists of standard, low-gradient, usually S-band, linac with beam energy from few to about ten $\mathrm{MeVs}$, and average beam power in the range of few watts to $100 \mathrm{~kW}$. As a rule these system are rather expensive, bulky, heavy, and not portable. The MicroLinac technology originally developed at SLAC [1] employs a compact Xband linear accelerator powered by an inexpensive, low power, pulsed magnetron [2,3]. However, to make the MicroLinac concept suitable for wider scope of applications, the conventional linac technology needs to be significantly advanced further to reduce cost, weight, and dimensions.

A considerable step forward has been made in development of compact X-band MicroLinacs utilizing high-impedance, all-copper, multi-cell structures [2,4]. A biperiodic part of a MicroLinac structure employs side [4] or on-axis [2] coupling cells enabling sufficient bandwidth and substantial number of cells sufficient to achieve beam energies exceeding $1 \mathrm{MeV}$ within a single section at limited power supply (sub-MW in X-band). However, fabrication of a multi-cell, tapered MicroLinac structure remains rather expensive and time consuming. It requires significant efforts to machine the large number of different cells with very high precision. Besides, each cell usually needs to be cold-tested, some of the cells may require remachining. After the clamped assembly is cold-tested, a multi-step brazing is performed followed by individual tuning of the cells of the brazed assembly.

An attractive opportunity for eased fabrication of MicroLinac is using of dielectric loaded structures, in which a smooth ceramic-lined metal tube replaces the metal periodic structure $[5,6,13]$. However, a wide practical implementation of this approach is prevented by a number of 
24 problems. Among them are single-wall multipactor [13] and charging of the dielectric material 25 capable to damage the material at practical values of pulse repetition rates and beam currents. In 26 a MicroLinac these problems are convoluted by substantial beam loss at finite capture of a 27 usually continuous beam injected from a thermionic injector at low energies (a few tens of keV). 28 An additional problem is a large spread of phase velocities required for a MicroLinac lying in the 29 range of (0.3-1)c. Shunt impedance over Q of a dielectric loaded structure at low phase velocity is noticeably lower compared to a conventional $\pi$-mode, iris loaded structure. In addition that

31 low phase velocity implies high permittivity for dielectrics $\left(\varepsilon_{\mathrm{r}}>20\right)$, which is usually associated 32 with elevated loss factors and thus further reduction of shunt impedance.

One interesting modification of a conventional iris (or disk) loaded traveling wave structure is related to a hybrid dielectric and iris loaded periodic accelerating structure [7]. That modification enables remarkably low (approaching to unity) overvoltage (defined as a ratio of maximum surface field to peak accelerating field) at still substantial shunt impedance and group

37 velocity. However, this modification does not take advantage of magnetic energy stored in the dielectric to provide magnetic coupling. More recently a modified dielectric loaded, periodic structure has been introduced to enhance coupling impedance [8]. However, that dielectric-structured approach seems to be difficult to 41 apply for fabrication of a long, multi-cell, strongly tapered MicroLinac. It may still suffer from 42 the issues related to dielectric exposition to the beam and multipactor similar to that in conventional dielectric-loaded and iris-loaded hybrid structures.

In this paper we introduce another modification of a hybrid metal-dielectric, periodic 45 slow-wave structure enabling higher group velocities, mitigation of the charging problem, and 46 potential simplification of structure prodiction for a low-current, low-gradient applications. 
47 In the next section we present the concept of the structure elementary cell using several examples

48 with different phase velocities and dielectric configurations. In Section 3 we analyze main

49 electromagnetic parameters of the structure. The electromagnetic simulations presented below

50 have been performed with the CST Studio Suite ${ }^{\mathrm{TM}}$ software [9].

\section{Disk-and-ring and disk-and-rod (DaR) slow wave structures}

Andreev's disk-and-washer (DaW) structure [10] and one of its variant [11] (sometimes called plane wave transformer, PWT) can be modified to build an inexpensive and compact, cmwave linac (MicroLinac). Indeed, in DaW classic structure one can eliminate the supporting rods by replacing them with dielectric support (e.g., rings or rods) and simultaneously replace the washers with a smooth-wall, continuous metal pipe. That would eliminate need in multi-cell brazing and possibly simplify the assembling. At the same time one can still try to ensure the compensated (biperiodic-like) electrodynamic behavior of the structure provided the dielectric supporting structure stores sufficient amount of magnetic energy and resonant frequencies of the operating and coupling (odd and even) modes are close enough for the same $\theta=\pi$ phase advance per cell. Examples of such disk-and-ring and disk-and-rod hybrid (dielectric-metal) slow wave structures are given in Fig. 1 and Fig. 4 respectively. The four slots on the disk edge seen in Fig. 1b provide vacuum conductivity to mitigate virtual leaks and enhance magnetic RF coupling. Field configurations shown in Fig. 2, Fig. 3, and Fig. 4 indicate the compensation effect. The

67 even mode employed in Disk-and-Ring (DaR) structure is TM01 mode unlike TM02 mode in Andreev's DaW structure. Along with using of high dielectric constant inserts that significantly 


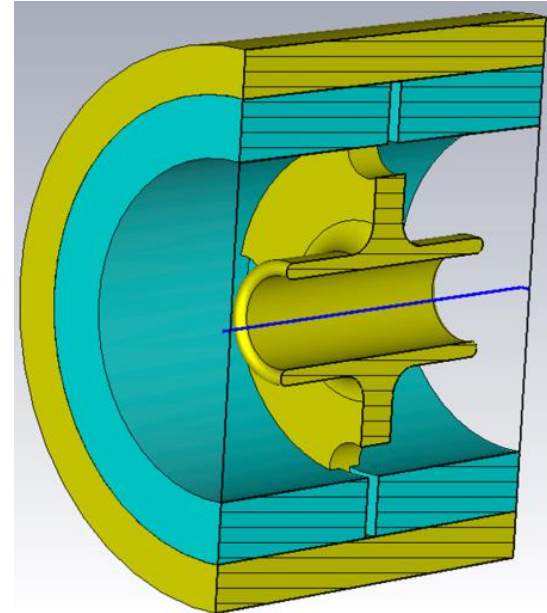

a)

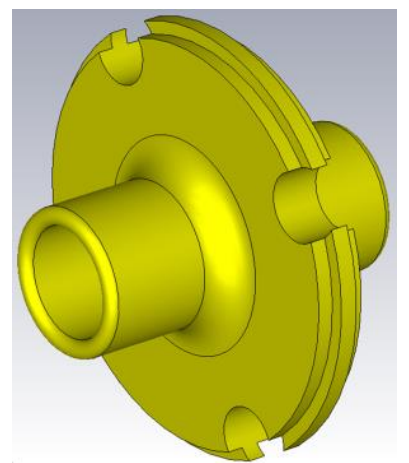

b)

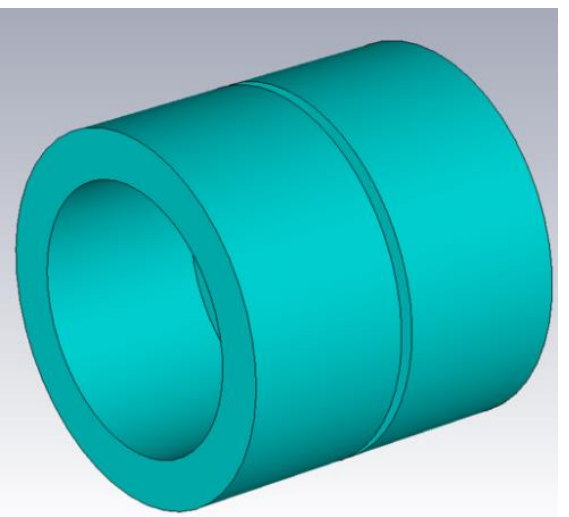

c)

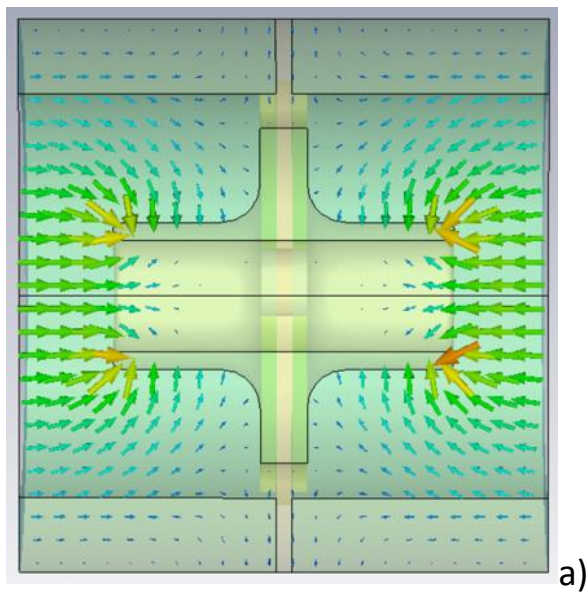
$\varnothing 3.175 \mathrm{~mm}$.

a)

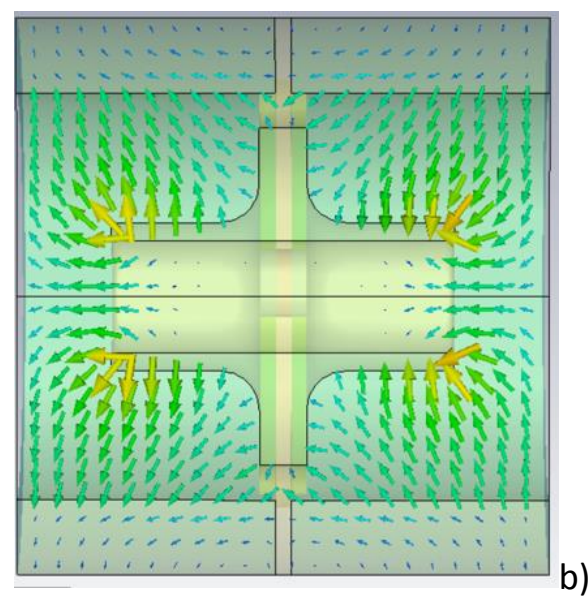
impedance possesses substantial overvoltage. The high electric field occurs on the edge of the 81

Fig. 2. RF electrical field map in plane of symmetry of the DaR cell of Fig. 1 for odd (a) and even (b) modes for $9.4 \mathrm{GHz}$ frequency and $180^{\circ}$ phase advance. The DaR structure example of Fig. 1 with re-entrant disks optimized for maximum shunt drift tube aperture resulting in up to 3.8 overvoltage. Maximum surface electric field is limited

Fig. 1. Disk-and-ring cell cut view (a) with disk (b) and Alumina or Sapphire rings (c) designed for $9.4 \mathrm{GHz}$ frequency, normalized phase velocity $\beta_{p h}=0.94 \mathrm{using}$ standard 0.75" OD, 0.62" ID copper tubing. Cell length is $15 \mathrm{~mm}$, beam aperture is 
82 by RF breakdown. For X-band the maximum RF field on a copper surface is about $110 \mathrm{MV} / \mathrm{m}$

83 for $800 \mathrm{~ns}$ pulse length [12]. That limits the peak accelerating field to about $29 \mathrm{MV} / \mathrm{m}$, which is

84 still more than sufficient for low power, low gradient MicroLinac applications. Note, the peak

85 electric field on the dielectric surface of a DaR structure is much lower than that in conventional

86 dielectric-filled tubular structures. That may facilitate the issues related to dielectric-enhanced

87 multipactor driving RF breakdown.

Simulations indicate unloaded Q-factor for the DaR structure does not exceed Q $\approx 4200$

89 with a good quality Alumina rings (see Fig. 7c). It means that in a cavity variant the structure

90 filling time will be limited by about $220 \mathrm{~ns}$ and pulse length can be as low as $700 \mathrm{~ns}$ or less. In a

91 traveling wave variant the pulse length can be reduced further due to the large group velocity.

92 That means higher accelerating gradients can be attained due to higher surface breakdown

93 thresholds at shorter pulses [12] if sufficiently strong dielectric is used.

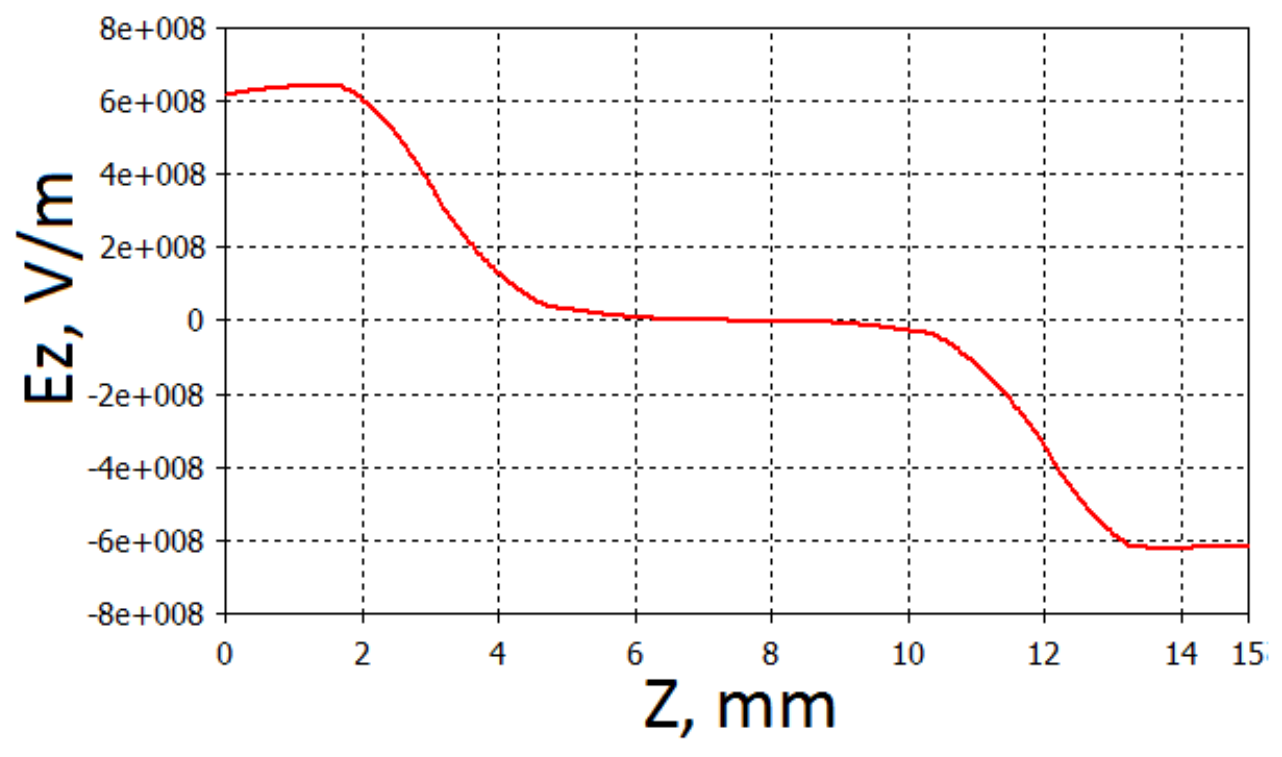

a) 


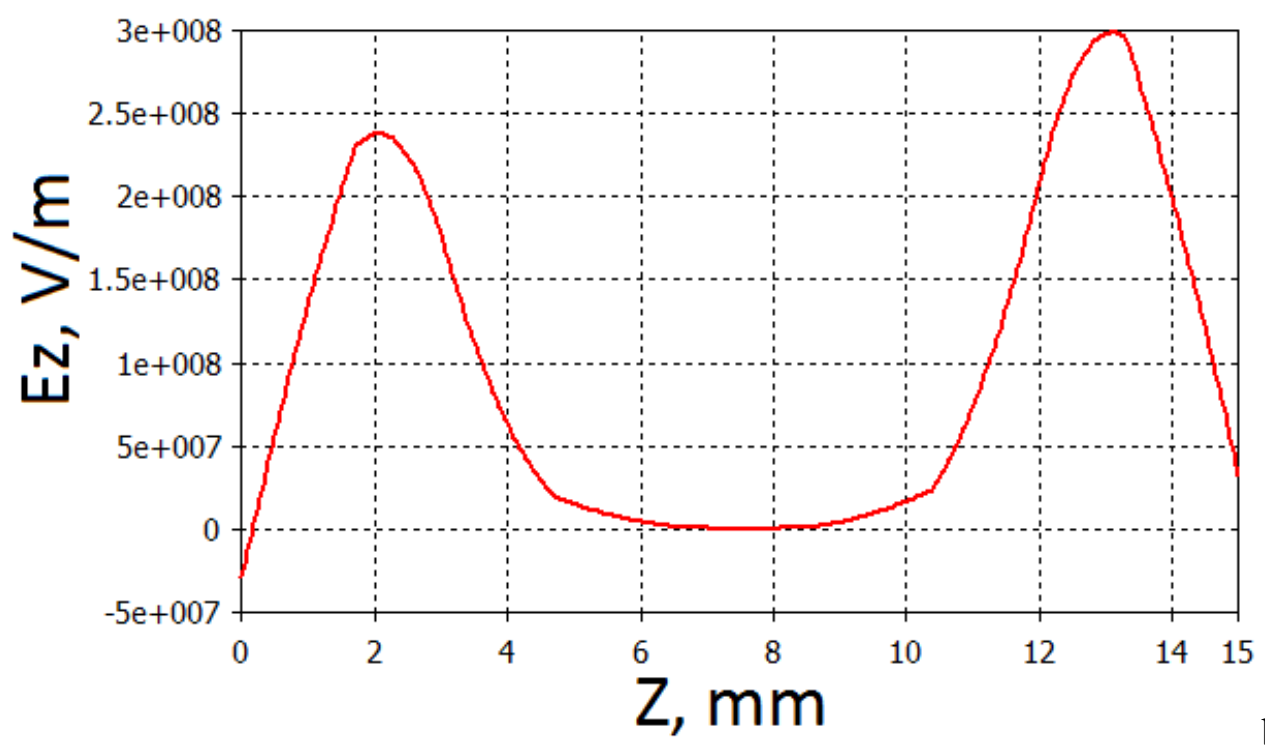

b)

96

97

Fig. 3. Longitudinal electric field plotted along the DaR cell for odd (a) and even (b) modes for $1 \mathrm{~J}$ stored energy at $9.4 \mathrm{GHz}$ frequency and $180^{\circ}$ phase advance.

The nature of asymmetry seen in Fig. $3 \mathrm{~b}$ is related to strong coupling between the even and odd TE01 modes at resonance in presence of numerical noise.

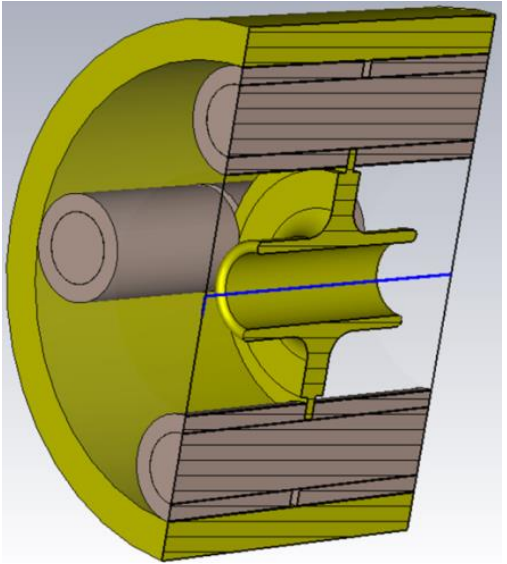

a)
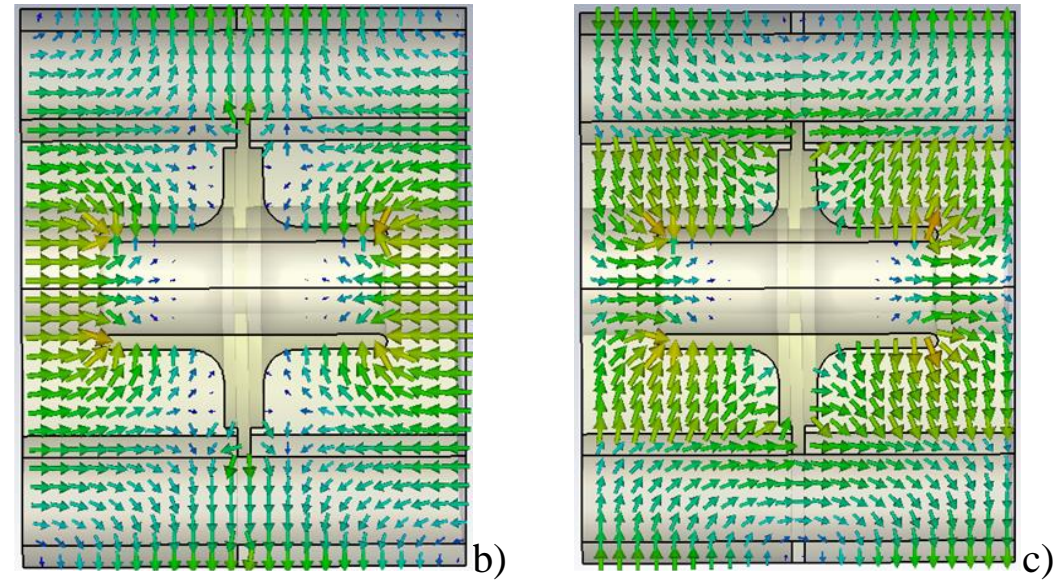

Fig. 4. Disk-and-rod cell cut view with Alumina (or Sapphire) rings (a) designed for $\sim 8.9 \mathrm{GHz}$ frequency, normalized phase velocity $\beta_{p h}=0.94$ using standard $1 " O D$, 0.75" ID copper tubing. Odd (b) and even (c) modes are shown. Shunt impedance of the cell is $0.72 \mathrm{M} \Omega$ for $\tan \delta=0.0001$.

One can see from the simulation results that for low loss dielectrics the shunt impedance can be made substantial and comparable to that of conventional disk loaded structure of X-band MicroLinac [4]. 


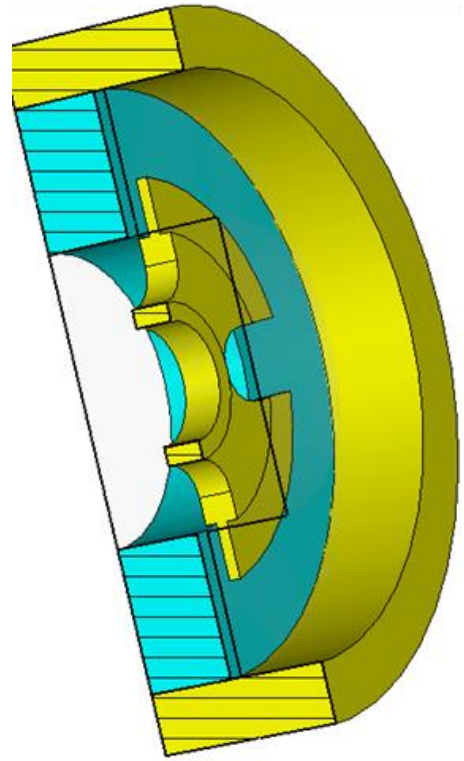

a)
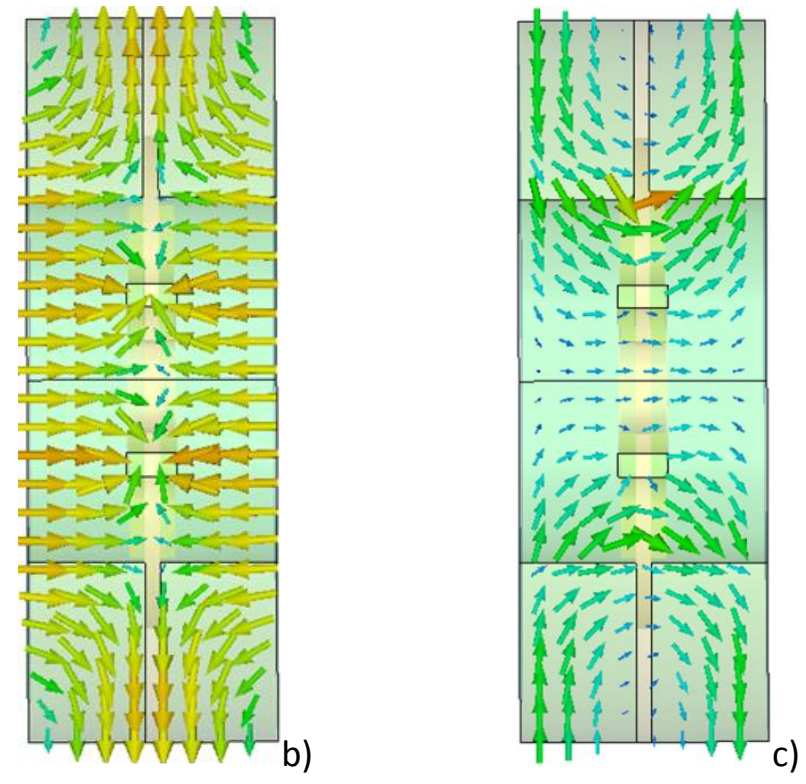

Fig. 5. Disk-and-Ring cell cut view with MgCaTiO3 rings $(\varepsilon=20$, one of the rings hidden) (a) designed for $8 \mathrm{GHz}$ frequency, normalized phase velocity $\beta_{p h}=0.34$ using standard 0.75" outer and 0.62" inner diameter copper tubing. Odd (b) and even (c) modes are shown.

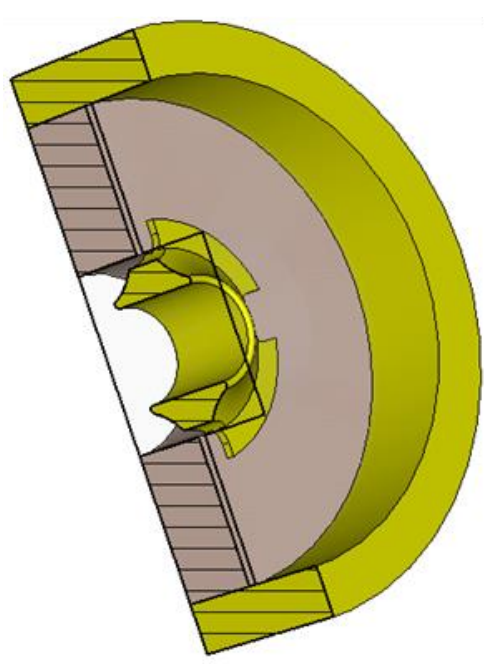

a)

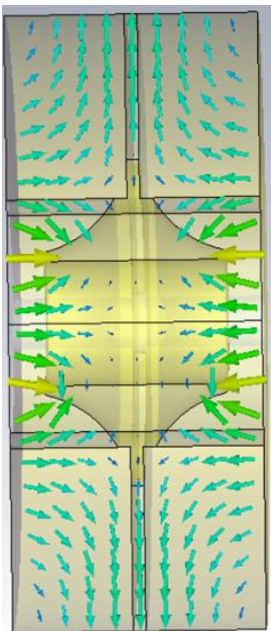

b)

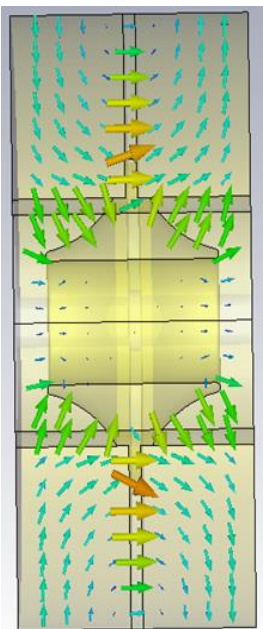

c) 
Fig. 6. Disk-and-Ring cell cut view with Alumina (or Sapphire) rings with one of the rings hidden (a) designed for $9.4 \mathrm{GHz}$ frequency, normalized phase velocity $\boldsymbol{\beta}_{p h}=\mathbf{0 . 4 1}$ using standard 0.75" OD, 0.62" ID copper tubing. Shunt impedance of the cell is $0.061 \mathrm{M} \Omega$ for $\tan \delta=0.0001$. Odd (b) and even (c) modes are shown.

\section{Dispersion, group velocity, shunt impedance, and Q-factor of DaR structure}

We consider here electrodynamic properties of the DaR structure in two variants: when the structure is perfectly tuned to the same frequency for both even and odd modes and substantially detuned. In Fig. 7 we show frequency dispersion, shunt impedance, and Q-factor plotted as a function of phase advance per cell shown in Fig. 1 for two TE01 modes resonant at $f=9.4 \mathrm{GHz}$ frequency and $\theta=\pi$ mode. From Fig. 7a one can clearly see that that at the resonant frequency mode "switching" occurs: the mode effectively interacting with the beam changes from that one having lower frequency at $f \leq 9.4 \mathrm{GHz}$ to the mode having higher frequency at $f>9.4$ GHz.

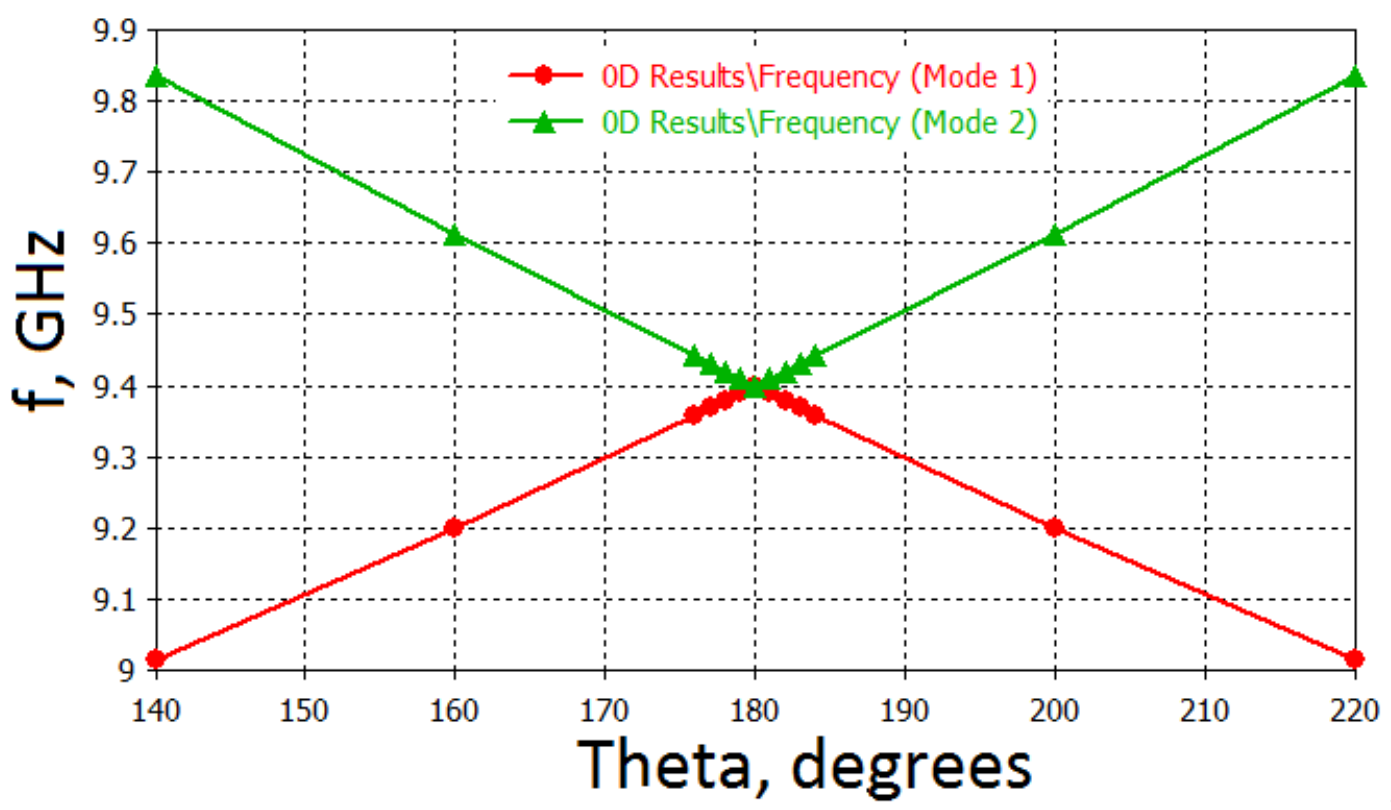




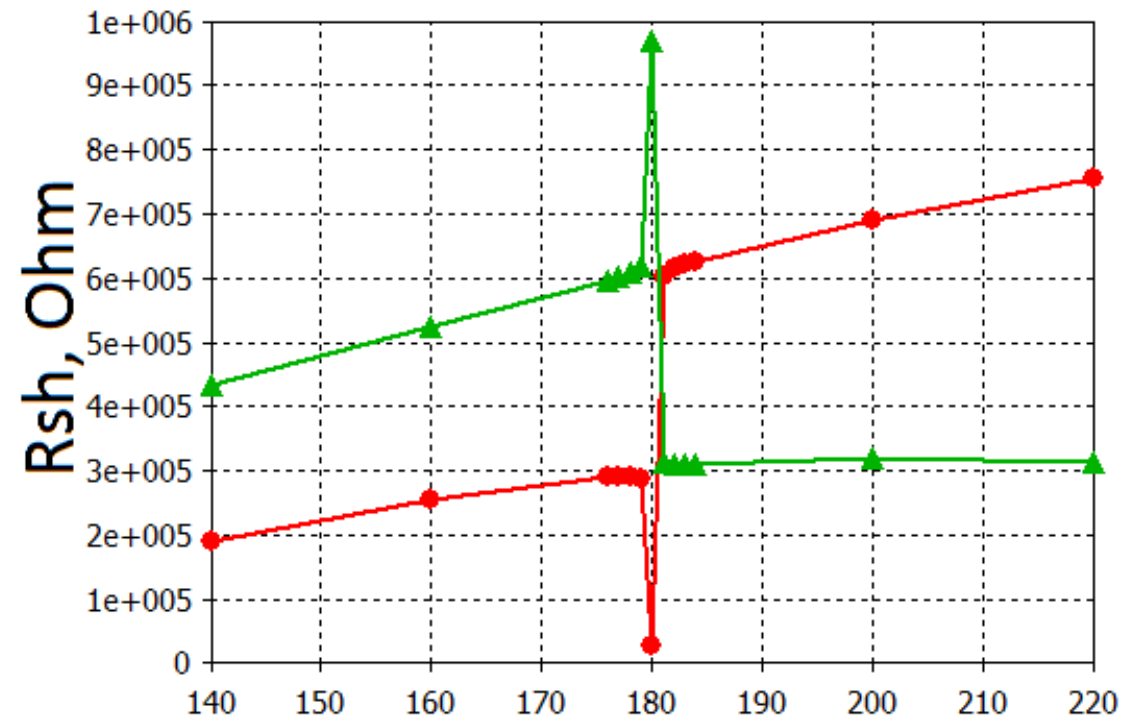

Theta, degrees

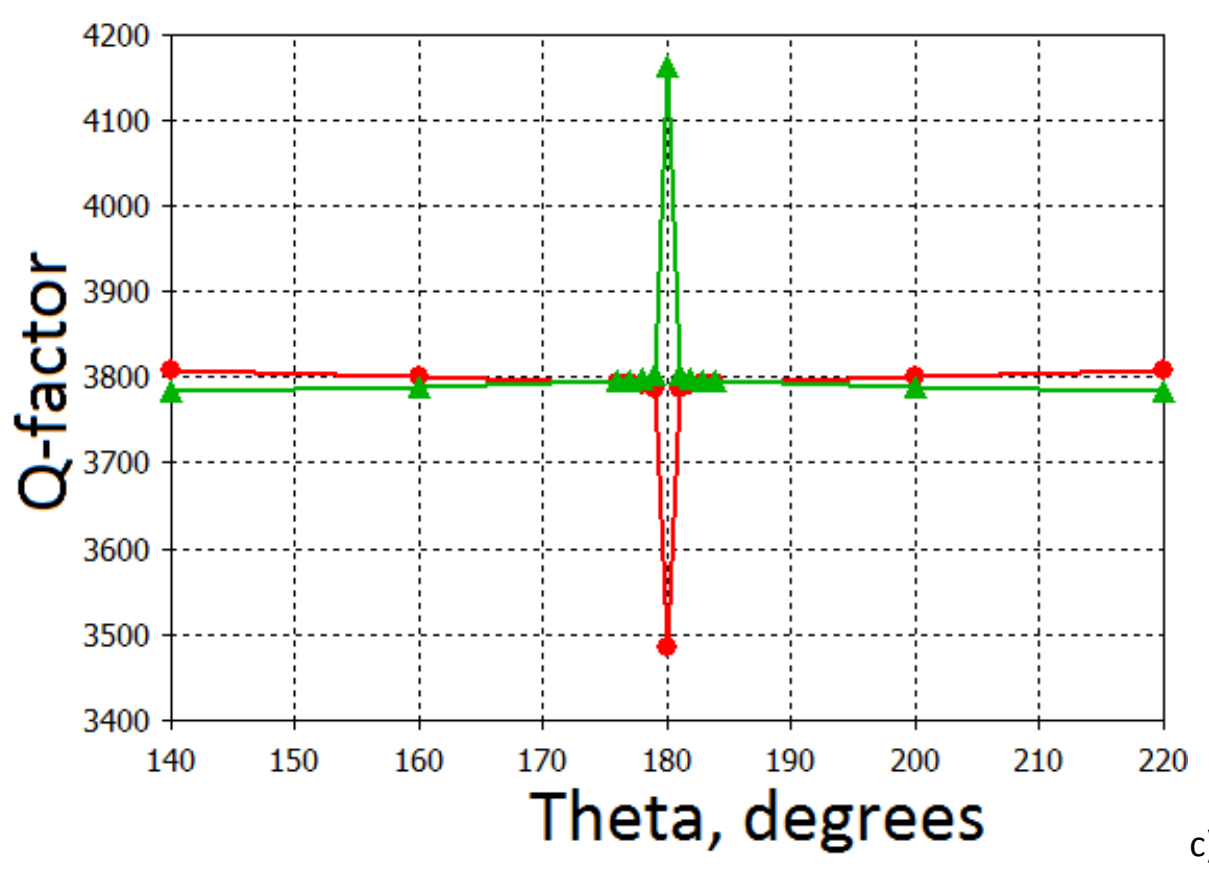

Fig. 7. Resonant frequency (a), shunt impedance (b), and Q-factor (c) of the DaR cell of Fig. 1 for $0.94 c$ phase velocity for even and odd modes as a function of phase advance. Alumina high grade ceramic is used with $\varepsilon=9.9$ and $\tan \delta=0.0001$. Red color is related to lower frequency mode, green color is related to higher frequency TE01 mode. Frequency difference at $\pi$-mode resonance is $0.5 \mathrm{MHz}$.

Next we have simulated the detuned structure by means of using a lower grade Alumina 
$139 \tan \delta=0.0019$ (instead of $\tan \delta=0.0001)$. No structure geometry optimization or other change has

140 been made for that variant. The eigenmode simulation results are presented in Fig. 8 showing 141 frequency dispersion, shunt impedance, and Q-factor plotted as a function of the phase advance 142 between the cell left and right boundaries. If we select smoothed part of the dispersion branches 143 of Fig. 8a curves, one can conclude that effective mode of operation corresponds to $\sim \pi / 2$ mode 144 for the center of phase advance range simulated. That full $2 \pi$ range for DaR structure appears as $145 \pi$ range for a conventional structure having twice less period. This is possible only if a virtual 146 coupling cell is involved. Therefore we can conclude that the DaR structure behaves 147 phenomenologically as a biperiodic compensated structure. Similarly to Fig. 7 the selection of 148 the dispersion curve branches can be validated again with the shunt impedance plots of Fig. 8b: 149 beam interacts with the higher frequency mode at $\theta<\pi$ (i.e. higher than the synchronous or 150 nominal phase velocity), and with lower frequency mode at $\theta>\pi$ (i.e. at lower than the resonant 151 phase velocity). Thus interaction pattern with the beam at $\theta=\pi$ crossing did not change even at 152 that significant $6 \%$ change in the dielectric constant compared to that of Fig. 7 . That means the 153 structure performance may remain intact (i.e. compensated). That indicates certain robustness in 154 terms of dielectric constant deviations and fabrication errors. 


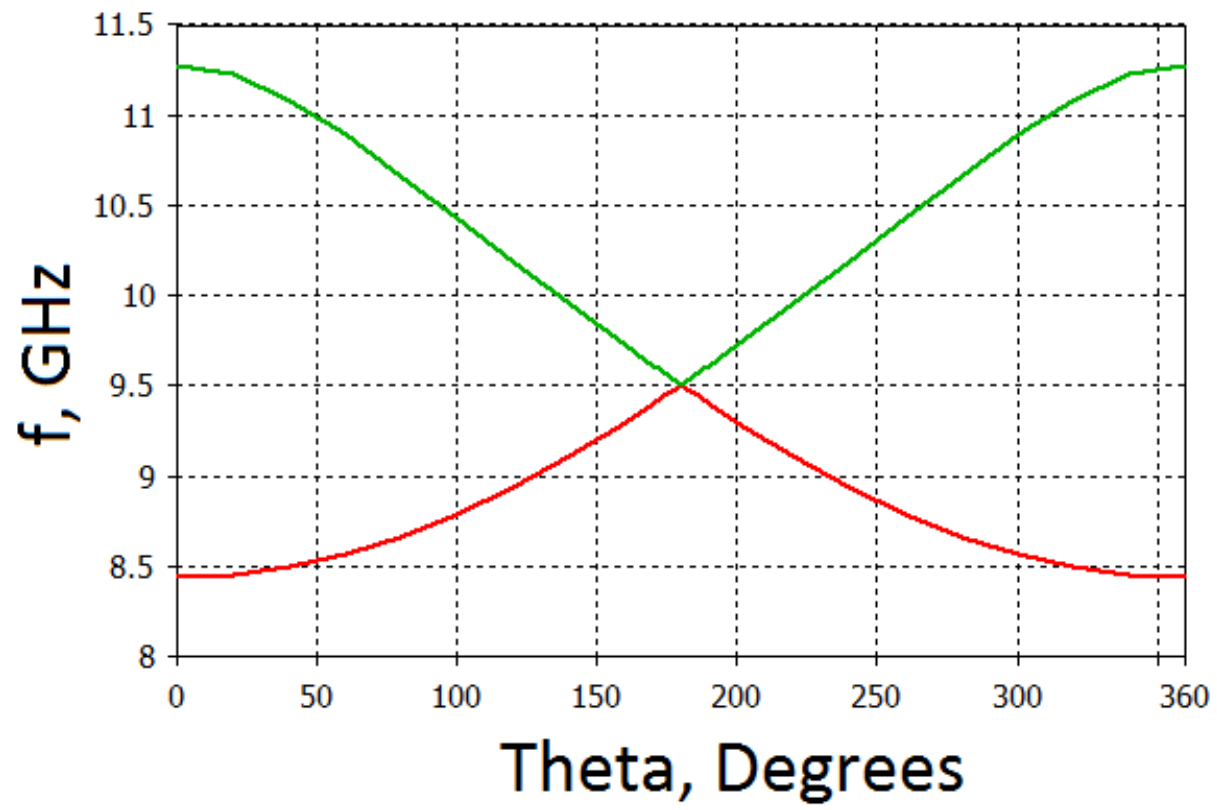

a)

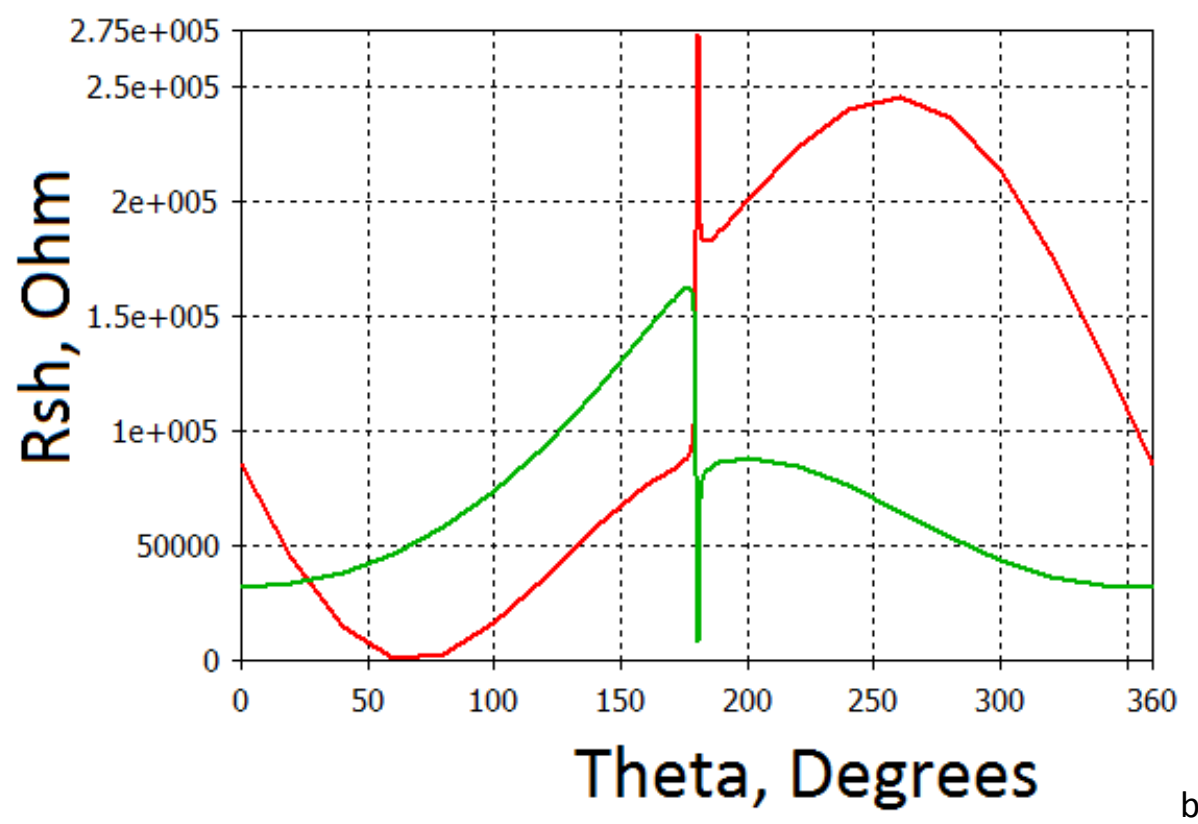


158

159

160

161

162

163

164

165

166

167

168

169

170 171 peak fields and lower RF energies per pulse; and e) capability of combining compensated $172\left(\left|\beta_{g r}\right|>0.05\right)$ and uncompensated $\left(\beta_{g r} \approx 0\right)$ cells in the same $\pi$ mode structure assembly. c)
Fig. 8. Resonant frequency (a), shunt impedance (b), and Q-factor (c) for the DaR cell of Fig. 1.

For DaR cells designed and optimized for phase velocities $\beta_{p h}$ varying in the range $0.34-0.94$ at the $f=9.4 \mathrm{GHz}$ resonant frequency we found from the simulations that the normalized group velocity $\beta_{g r}$ varies from -0.19 to -0.1 for $\varnothing 0.62$ " internal diameter of the external copper pipe. That indicates strong magnetic coupling opening great opportunity for multi-cell performance of the assembly employed as a standing wave (SW) linac.

In general such a high absolute value of group velocity may offer a number of benefits: a) significantly reduced sensitivity to fabrication imperfections; b) a large number of cells in a single SW section and/or increased frequency separation between adjacent modes; c) elimination of power flow effect distorting resonant frequencies of the cells and related field profile along the section; d) capability to operate of short pulse lengths in a traveling wave design at higher

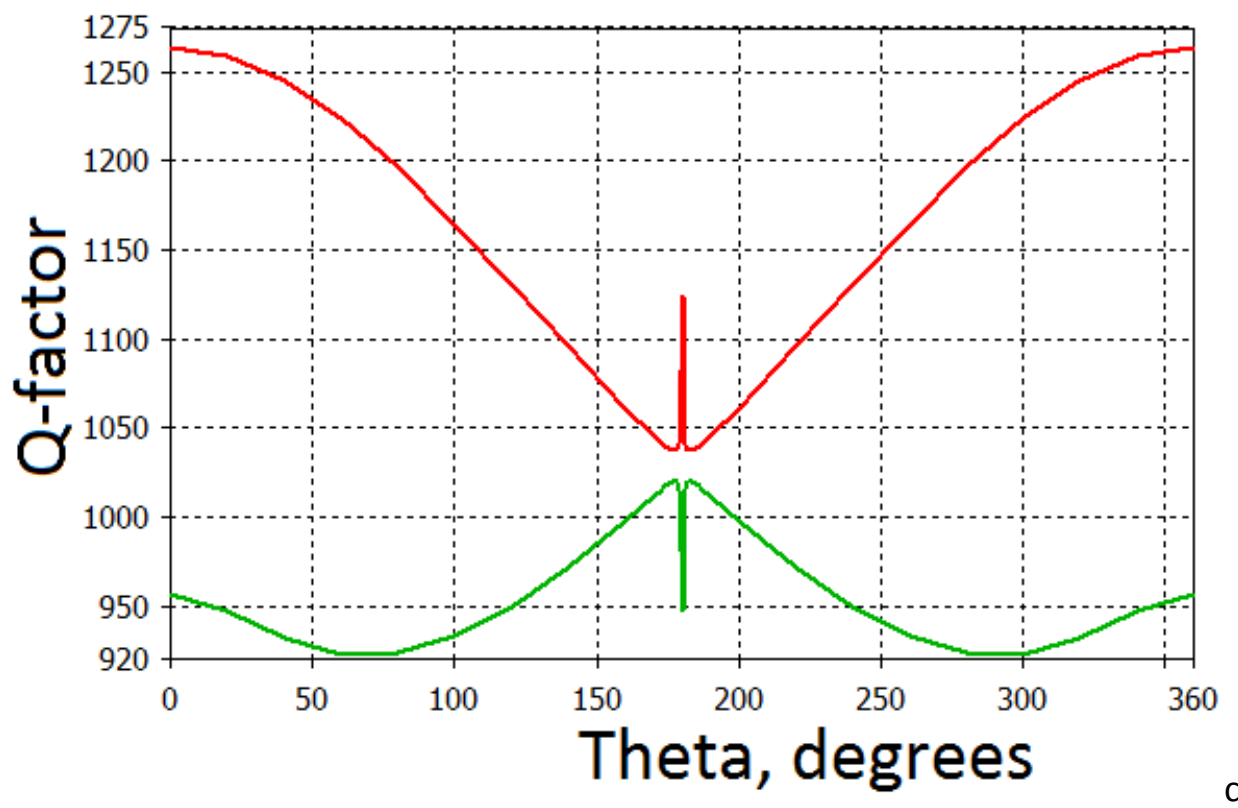




\section{Discussion}

175 We introduced here an elementary cell that can be used to build a novel type of hybrid, 176 dielectric-metal, periodic structure combining substantial shunt impedance and potential for 177 eased fabrication. Unlike disk loaded hybrid structure [7] the coupling is of magnetic type. 178 Similar to Andreev's DaW structure the DaR structure exhibits $\pi / 2$ effective (electrodynamic) 179 operating mode (while "geometric" $\pi$ mode for the cell), because the radial gap between the disk 180 periphery and the tube housing may act as a resonantly coupled cell.

The dielectric part of the structure is somewhat isolated by the metal disks from the direct

182 charging by the beam halo. Nevertheless the charging by the beam may affect the performance.

183 That effect is expected to be dominated by charging of the disks. That can be mitigated by using 184 a thin wire (pin) placed between the disk and the tubular housing or a thin conducting coating of 185 the dielectric parts with a metal film having thickness much smaller than the skin depth (e.g., 186 nanometers vs. $700 \mathrm{~nm}$ skin depth in Copper in X-band). Note, unlike the TiN coating used for 187 multipactor suppression [13] the main function of that coating is removal of static charge 188 accumulated mostly on the disks.

189 The structure can in principle be assembled as a stack of non-brazed cells in a tubular 190 housing. Therefore it can be considered as a candidate for, e,g., inexpensive mass-production of 191 MicroLinacs capable of replacing radioactive sources. It also offers a certain potential for 192 racetrack microtrons, traveling wave tubes, wakefield radiators (harmonic extractors [14]), and 193 RF deflectors employed for phase space manipulation, beam diagnostics, and material 194 spectroscopy with ultrafast electron diffraction. 


\section{Acknowledgements}

197 This work was supported by the U.S. Department of Energy (award No. DE-SC-FOA-0011370).

198 The authors are grateful to Dr. Arden Dougan for her interest to that development. The authors

appreciate Dr. Sergey Kutsaev for validating simulations, Josiah Hartzell for initial mock-up

Murokh, Robert Berry and Ronald Agustsson for discussions related to performance, packaging,

fabrication and engineering of a corresponding MicroLinac system.

\section{REFERENCES}

[1] N. Ackerman et al., "MicroLinac - A Portable Accelerator for Radiography", The 12th Advanced Accelerator Concepts Workshop, 2006.

[2] T. Yamamoto, T. Natsui, F. Sakamoto, M. Uesaka, N. Nakamura and E. Tanabe, "Development of portable X-band linac X-ray source for non-destructive testing", Proceedings of the Joint International Workshop: Nuclear Technology and Society-Needs for Next Generation, Berkeley, California (Berkeley, California, 2008).

[3] S. Boucher, X. Ding, A. Murokh, In Proceedings of Intern. Particle Accelerator Conf. (IPAC'10, Kyoto, Japan, 2010) 178.

[4] S. Boucher, R. Agustsson, L. Faillace, J. Hartzell, A. Murokh, A. Smirnov, S. Storms, K. Woods. Proceedings of Intern. Particle Accelerator Conf. (IPAC2013, Shanghai, China, 2013) 3746.

[5] E. Chojnacki et al., J. Appl. Phys.69 (1991) 6257

[6] P. Zou et al., Rev. Sci. Instrum.71, (2000) 2301-2304.

[7] P. Zou, L. Xiao, X. Sun, W. Gai. Proceedings of the 2001 Particle Accelerator Conf., (Chicago, PAC 2001) 3966.

[8] D. Satoh, M. Yoshida, N. Hayashizaki. Phys. Rev. Accelerators and Beams 19, 011302 (2016).

[9] https://www.cst.com/2015

[10] V. G. Andreev et al., Proc. Intern. Conf. on High Energy Accelerator, (Frascati, Italy, 1965); Soviet Physics - Technical Physics 13 (1969) 1070.

[11] R. Zhang, P. Davis, G. Hairapetian, M. Hogan, C. Joshi, M. Lampel, S. Park, C. Pellegrini, J. Rosenzweig and G. Travish. IEEE Proc. of Particle Accelerator Conf. (1996) 1102

[12] S. Döbert. RF-Breakdown in High-Frequency Accelerators. Stanford Linear Accelerator Center, SLAC-PUB-10463, 2004.

[13] C. Jing, J. G. Power, R. Konecny, W. Gai, S.H. Gold, A. K. Kinkead. In Proceedings of 2005 Particle Accelerator Conference, Knoxville, Tennessee (2005) 1566 
[14] D. Newsham, A. Smirnov, D. Yu, W. Gai, R. Konecny, W. Liu, H. Braun, G. Carron, S. Doebert, L. Thorndahl, I. Wilson, W. Wuensch, in Proc. of 2003 Part. Acc. Conf., (Portland, Oregon, May 12-16, 2003) 1156. 